INFLAMMATORY BOWEL DISEASE

\title{
Once daily high dose probiotic therapy (VSL\#3) for maintaining remission in recurrent or refractory pouchitis
}

\author{
T Mimura, F Rizzello, U Helwig, G Poggioli, S Schreiber, I C Talbot, R J Nicholls, P Gionchetti, \\ M Campieri, M A Kamm
}

Gut 2004;53:108-114

See end of article for authors' affiliations

Correspondence to: Professor M A Kamm, St Mark's Hospital, Watford Rd, Harrow, Middlesex HAl 3UJ, UK; kamm@ic.ac.uk

Accepted for publication 5 August 2003
Background: Ten to $15 \%$ of patients with pouchitis experience refractory or recurrent disease. The aim of this study was to evaluate the effectiveness of a single daily high dose probiotic preparation (VSL\#3) in maintaining antibiotic induced remission, and quality of life (QOL), for one year in such patients.

Methods: Patients with pouchitis at least twice in the previous year or requiring continuous antibiotics, associated with a pouchitis disease activity index (PDAl) $\geqslant 7(0=$ perfect; $18=$ worst), in whom remission was induced by four weeks of combined metronidazole and ciprofloxacin, were randomised to receive VSL\#3 6 g or placebo once daily for one year or until relapse. Symptomatic, endoscopic, and histological evaluations were made before, and two and 12 months after randomisation or at the time of relapse. Remission was defined as a clinical PDAI $\leqslant 2$ and endoscopic PDAI $\leqslant 1$. Relapse was defined as an increased clinical PDAl score $\geqslant 2$ and increased endoscopic PDAl score $\geqslant 3$. QOL was assessed using the inflammatory bowel disease questionnaire (IBDQ).

Results: Thirty six patients were randomised: 20 to VSL\#3 and 16 to placebo. Remission was maintained at one year in 17 patients $(85 \%)$ on VSL\#3 and in one patient $(6 \%)$ on placebo $(p<0.0001)$. The IBDQ score remained high in the VSL\#3 group $(p=0.3)$ but deteriorated in the placebo group $(p=0.0005)$. Conclusion: The once daily high dose probiotic VSL\#3 is effective in maintaining antibiotic introduced remission for at least a year in patients with recurrent or refractory pouchitis. This is associated with a high level of quality of life.
$\mathrm{T}$ en to $20 \%$ of patients with ulcerative colitis still undergo colectomy because of refractory inflammation or malignant change of the mucosa. Restorative proctocolectomy with ileal pouch anal anastomosis (IPAA) is now the commonest operation for ulcerative colitis. ${ }^{12}$ However, patients having this procedure experience more short and long term complications than those with a permanent ileostomy. ${ }^{2} 3$ "Pouchitis" is one of the more common long term complications and is specific to this procedure.

Pouchitis is a non-specific acute inflammation within an ileal reservoir, leading to increased frequency with loose stool and abdominal cramping. The cumulative incidence of pouchitis varies from 20 to $50 \%$, depending on the definition and length of follow up. ${ }^{3-8}$ When followed for more than 10 years, approximately half experience pouchitis at least once. ${ }^{7}$

Antibiotic therapy is often effective, but $5-15 \%$ of patients with an IPAA created for ulcerative colitis experience "refractory or frequently recurrent" pouchitis.. ${ }^{468}$ These patients experience relapsing recurrent pouchitis or chronic persistent pouchitis (fig 1). Frequently relapsing pouchitis is usually regarded as more than two episodes during one year, after a reasonable response to therapy. Chronic pouchitis can be divided into "treatment responsive" and "treatment resistant". Patients with treatment responsive chronic pouchitis respond to some therapy, but when therapy is stopped, pouchitis relapses. Patients therefore either have to continue drug therapy or endure their symptoms. Patients with treatment resistant pouchitis do not respond to conventional therapy and continue to suffer symptoms.

Even patients who have not responded to standard short courses of single antibiotics ${ }^{9}$ are likely to respond to more intense antibiotic therapy. We recently reported in an open study that a prolonged course of a combination of metronidazole and ciprofloxacin is effective in treating recurrent and refractory pouchitis, with an associated improvement in quality of life. ${ }^{10}$ In that study, $36(82 \%)$ of 44 patients with recurrent or refractory pouchitis went into remission after a four week course of metronidazole and ciprofloxacin.

Once the inflammation has been reduced or eliminated, the problem of maintaining remission remains. Modification of the bacterial flora, using a "probiotic" preparation, may be helpful. Probiotics encompass live bacteria belonging to natural bacterial flora without pathogenicity which are thought to exert health benefits beyond inherent basic nutrition. They comprise a variety of bacterial species, including lactobacilli, bifidobacteria, and streptococci, as well as non-pathogenic Escherichia coli.

A previous study from part of our group has demonstrated that twice daily probiotic ingestion will maintain remission in a majority of patients with previous pouchitis over a nine month period. In that study, 40 patients with refractory pouchitis were initially treated with a combination of ciprofloxacin and rifaximin for 15 days. After induction of remission, 20 patients were randomised to receive VSL\#3 $3 \mathrm{~g}$ twice per day and 20 received placebo, for nine months or until relapse. Seventeen patients on VSL\#3 remained in remission while all on placebo relapsed. ${ }^{11}$

Response to treatment of the primary condition may not always mirror improvement in patient well being. This may relate to the nature of the treatment or other unrecognised factors being the main source of morbidity. For these reasons health related quality of life (QOL) assessment is increasingly important when the effect of any therapy is evaluated in

Abbreviations: IPAA, ileal pouch anal anastomosis; $Q O L$, quality of life; IBDQ, inflammatory bowel disease questionnaire; PDAl, pouchitis disease activity index 


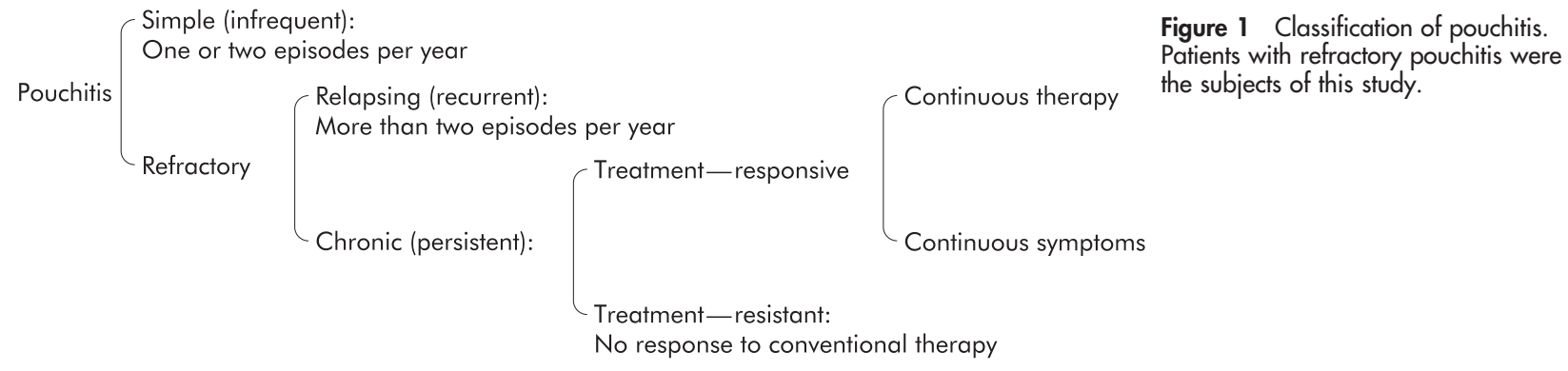

clinical trials and clinical management. The inflammatory bowel disease questionnaire (IBDQ) has been developed and validated to assess QOL for patients with inflammatory bowel disease. $^{12}{ }^{13}$ In a previous study, we demonstrated the sensitivity of the IBDQ to detect change in response to treatment in patients receiving therapy for pouchitis. ${ }^{10}$ As probiotics have not been extensively evaluated in relation to their positive and negative clinical effects, we therefore included QOL assessment in this study.

The aim of this study was to evaluate the clinical efficacy, and effect on QOL, of a probiotic preparation (VSL\#3) in maintaining remission in patients with recurrent or refractory pouchitis.

\section{PATIENTS AND METHODS}

\section{Patients}

This two centre study was approved by the ethics committee at both institutions. Written informed consent was obtained before participation.

The pouchitis disease activity index (PDAI) was used to assess disease activity. This index is a scoring system used to assess inflammation within the ileal pouch, and includes

\begin{tabular}{ll}
\hline Table 1 Pouchitis disease activity index & \\
\hline Criteria & Score \\
\hline Clinical & \\
Stool frequency & \\
Usual postoperative stool frequency & \\
$1-2$ stool/day > postoperative usual & 1 \\
$\geqslant 3$ stools/day > postoperative usual & 2 \\
Rectal bleeding & \\
None or rare & 0 \\
Present daily & 1 \\
Faecal urgency or abdominal cramps & \\
None & 0 \\
Occasional & 1 \\
Usual & 2 \\
Fever (temperature $>37.8^{\circ} \mathrm{C}$ ) & 0 \\
Absent & 1 \\
Present & \\
Endoscopic inflammation & \\
Oedema & 1 \\
Granularity & 1 \\
Friability & \\
Loss of vascular pattern & 1 \\
Mucous exudate & 1 \\
Ulceration & 1 \\
Acute histological inflammation & 1 \\
Polymorphonuclear leucocyte infiltration & \\
Mild & \\
Moderate+crypt abscess & \\
Severe+crypt abscess & \\
Ulceration per low power field (mean) & \\
<25\% & \\
$25-50 \%$ & 2 \\
$>50 \%$ & 3 \\
\hline & 1 \\
\hline
\end{tabular}

clinical, endoscopic, and acute histological criteria (table 1). ${ }^{14}$ Each criterion has six points, with the total score ranging from 0 (no inflammation) to 18 (worst inflammation).

Patients were included if they had suffered active refractory or recurrent pouchitis and successfully achieved remission after a four week course of combined metronidazole $400 \mathrm{mg}$ or $500 \mathrm{mg}$ twice daily and ciprofloxacin $500 \mathrm{mg}$ twice daily.

Active refractory or recurrent pouchitis was defined as a total PDAI score of $\geqslant 7$ (possible range $0=$ perfect to $18=$ worst) and a history of relapsing or persistent pouchitis, as described in fig 1. Remission was defined as both a clinical PDAI score $\leqslant 2$ (possible range $0=$ no symptoms to $6=$ worst symptoms) and endoscopic PDAI score $\leqslant 1$ (possible range $0=$ no inflammation to $6=$ worst inflammation).

\section{Randomisation and sample size}

Pharmacists at each institute were in charge of the randomisation. The results of the randomisation were known only to the pharmacists until the end of this study when the data were analysed.

Sample size was based on the estimate of $80 \%$ recurrence in the placebo group and $25 \%$ in the probiotic group over a one year period. These estimates followed relapse findings in a previous study by Gionchetti and colleagues. ${ }^{11}$ Eighteen patients were needed in each group to provide an $80 \%$ chance of a significant difference at the 0.05 level using a two tailed test.

Randomisation was performed separately in each institution. The number of patients to be recruited was targeted at 36 without setting the minimum or maximum number at each institution. To avoid a disproportionate number of patients in the active or placebo group at either institution, randomisation was performed in blocks of four (two placebo and two active) at each institution.

\section{Study medication and compliance}

The probiotic preparation used in this study was VSL\#3 (VSL pharmaceuticals Inc., Fort Lauderdale, USA). VSL\#3 $3 \mathrm{~g}$ sachets contain 300 billion bacteria/g, comprising four strains of lactobacilli (acidophilus, delbrueckii subsp bulgaricus, casei, plantarum), three strains of bifidobacteria (breve, longum, infantis), and one strain of Streptococcus salivarius subsp thermophilus. Placebo sachets contained maize.

Compliance was assessed by counting the dispensed and returned sachets, as well as by questioning patients. Compliance rate was calculated by dividing the number of taken sachets by that of dispensed sachets.

Medication consisted of either VSL\#3 $6 \mathrm{~g}$ once daily in the evening or identical appearing placebo sachets. Patients took the sachets for 12 months, provided their pouchitis did not relapse.

Evaluation of disease activity and quality of life Symptoms were assessed and physical examination performed immediately prior to randomisation and every two months for 12 months or until relapse. 
Endoscopic and histological evaluations were made before and two months after randomisation, and then at 12 months or at relapse. Grading was according to the PDAI. For endoscopic evaluation, one doctor at each institution (PG at Bologna; TM at St Mark's) performed a flexible sigmoidoscopy, during which two biopsies were taken for histological assessment of the extent of acute inflammation.

Relapse was defined as an increase in the clinical PDAI score of $\geqslant 2$ together with an increase in the endoscopic PDAI score of $\geqslant 3$ compared with the baseline score at remission (study entry) after antibiotic therapy.

The primary outcome of this study was the cumulative maintained remission rate at 12 months, by intention to treat analysis.

Health related QOL was a secondary outcome, and was assessed at study entry, every two months during the study, and at the time of relapse. The IBDQ assesses bowel, systemic, and emotional symptoms as well as social function. ${ }^{12}$ The IBDQ score ranges from 32 (worst quality of life) to 224 (best).

Patient's general satisfaction with the treatment was the other secondary outcome, and was evaluated at study entry and every two months, and at the time of relapse. Patients were asked "How satisfied, happy, or pleased during the last two weeks have you been with the treatment you have been taking?". Patients chose their answer from the following five options: (1) very dissatisfied, unhappy most of the time; (2) generally dissatisfied, unhappy; (3) neither dissatisfied nor satisfied; (4) generally satisfied, pleased; (5) very satisfied, happy most of the time.

\section{Stool bacterial assessment}

To confirm the presence of viable probiotic bacteria in the stool of patients taking the active preparation, stool analysis was undertaken in a subgroup of patients on active and placebo. Analysis was undertaken for Streptoccoccus thermophilus and Bifidobacterium. Analysis was undertaken without knowledge as to whether the subject was on active or placebo medication.

Faecal samples of 12 patients were examined at the beginning and after 60 days of VSL\#3 $(n=6)$ or placebo $(\mathrm{n}=6)$ treatment. Stool specimens were collected in sterile plastic containers, immediately stored at $-20^{\circ} \mathrm{C}$, and analysed within 10 days.

\section{PCR detection of Streptoccoccus thermophilus and Bifidobacterium}

Amplification reactions were carried out in a Biometra Thermal Cycler II (Biometra, Göttingen, Germany). Dynazyme II (Celbio, Milan, Italy) was used as a thermostable polymerase. The total volume of each reaction mixture was $25 \mathrm{ml}$ and cells from plate colonies were used directly as templates, without isolation of chromosomal DNA. Amplification of $S$ thermophilus was obtained using the primer set ThI/ThII developed by Timisjärvi and Alatossava in 1997. The reaction mixture consisted of $200 \mu \mathrm{M}$ of each dNTP, $1 \mu \mathrm{M}$ of ThI and ThII primer, and $1 \mathrm{U}$ of Dynazyme II. The amplification profile was $95^{\circ} \mathrm{C}$ for one minute, $55^{\circ} \mathrm{C}$ for 30 seconds, and $72^{\circ} \mathrm{C}$ for one minute. This was repeated for 40 cycles. The programme also included preincubation at $95^{\circ} \mathrm{C}$ for five minutes before the first cycle and a final incubation at $72^{\circ} \mathrm{C}$ for five minutes. Bifidobacteria amplification using the primer sets Bif164/Bif662 (Kok et al, 1996), InfY-BV.L/R and BreY-BV.R/L (Brigidi et al, 2000), specific for the genus Bifidobacterium and for the VSL-3 strains $B$ infantis Yl and $B$ breve Y8, respectively, was accomplished under the following experimental conditions. Enumeration of $S$ thermophilus, $B$ infantis Y1, and B breve Y 8 was performed by direct amplification of 30-50 colonies, randomly selected from the highest dilutions of ST modified and RB agar plates. Aliquots $(5-10 \mu \mathrm{l})$ of the amplified products were subjected to gel electrophoresis in $2 \%$ agarose gels and were visualised by ethidium bromide staining.

All results on bacterial analysis were expressed as mean (SD). Values of $\mathrm{p}<0.05$ were considered statistically significant.

\section{Safety assessment}

All unfavourable unexpected symptoms were recorded in a diary kept by patients during the study. Laboratory studies, including a complete blood count and blood chemistry measurements, were performed at study entry, after two and 12 months, and at the time of relapse.

\section{Statistical analysis}

Statistical analysis was performed using PrismTM version 2.0 (GraphPad Inc., San Diego, California, USA). For description of data, median and range were calculated. Fisher's exact probability test (two tailed) was used for qualitative data that could be expressed in a $2 \times 2$ contingency. The Mann-Whitney test (unpaired, two tailed) was used to compare quantitative data between the VSL\#3 and placebo treated groups at the time of study entry and at 12 months or relapse.

The cumulative maintained remission rate over the 12 month follow up period was assessed by Fisher's exact test, and the Kaplan-Meier method and log rank test to demonstrate the clinical course in relation to relapse over the 12 month period.

The Wilcoxon signed rank test (paired, two tailed) was used to compare the PDAI score, IBDQ score, and the patient satisfaction score at study entry and at the time of relapse or at 12 months in each group.

\section{RESULTS}

\section{Patient characteristics}

Thirty six patients (20 males; median 36 years $)$ with refractory or recurrent pouchitis were in remission after the antibiotic regimen and entered the study between May 1999 and June 2000, and were followed up until June 2001. At St Mark's, four patients had recurrent and three chronic pouchitis; in Bologna 22 patients had recurrent and seven chronic pouchitis. Twenty patients were randomised to receive VSL\#3 and 16 placebo. There were no significant differences between the two groups at the time of randomisation with regard to patient characteristics or PDAI and IBDQ scores (table 2). At entry, median PDAI score was 3 (range 1-7), clinical score 1 (0-2), endoscopic score l (0-1), and histological score $1(0-4)$ in the VSL\#3 group, compared with a PDAI score of 3 (range 1-4), clinical score 1 (0-2), endoscopic score $1(0-1)$, and histological score $1(0-2)$ in the placebo group.

All patients showed excellent compliance with the medication. Median compliance rate was $96 \%$ in the VSL\#3 and $97 \%$ in the placebo group, with no significant difference between the two groups.

\section{Relapse rate in each group}

As shown in fig 2, 17 of 20 patients on VSL\#3 were still in remission at 12 months. Two patients relapsed at two and eight months. One patient dropped out due to acute gastroenteritis-like symptoms. Of the 16 patients receiving placebo, only one remained in remission.

As presented in fig 3 as a Kaplan-Meier method, the cumulative maintained remission rate over the 12 month period was $85 \%$ in the VSL\#3 group and $6 \%$ in the placebo group $(\mathrm{p}<0.0001)$. Half of the patients on placebo relapsed within the first two months. 


\begin{tabular}{|lccl}
\hline Table 2 & Patient characteristics in the VSL\#3 and placebo groups & \\
\hline & VSL $\# \mathbf{3}(\mathbf{n}=20)$ & Placebo $(\mathbf{n}=16)$ & p Value \\
\hline Sex (M/F) & $12 / 8$ & $8 / 8$ & 0.74 \\
Age (y) & $36(22-64)$ & $36(27-53)$ & 0.97 \\
Age at UC onset (y) & $24(12-49)$ & $22(13-37)$ & 0.44 \\
Age at pouch surgery (y) & $33(14-60)$ & $31(17-51)$ & 0.76 \\
Time between ileostomy closure & $9.5(0.5-87)$ & $15(0.5-72)$ & 0.69 \\
and first pouchitis onset (months) & $26(9-168)$ & $33(10-118)$ & 0.34 \\
Time since the first pouchitis ever (months) & $14 / 6$ & $12 / 4$ & 1.0 \\
Clinical pattern of pouchitis (relapsing/chronic) & $0 / 20$ & $3 / 13$ & 0.08 \\
Extraintestinal manifestations (+/-) & $3(1-7)$ & $3(1-4)$ & 0.70 \\
PDAl score at randomisation & $189(104-212)$ & $169(154-215)$ & 0.38 \\
IBDQ score at randomisation & & \\
\hline UC, ulcerative colitis; PDAl, pouchitis disease activity index; IBDQ, inflammatory bowel disease questionnaire.
\end{tabular}

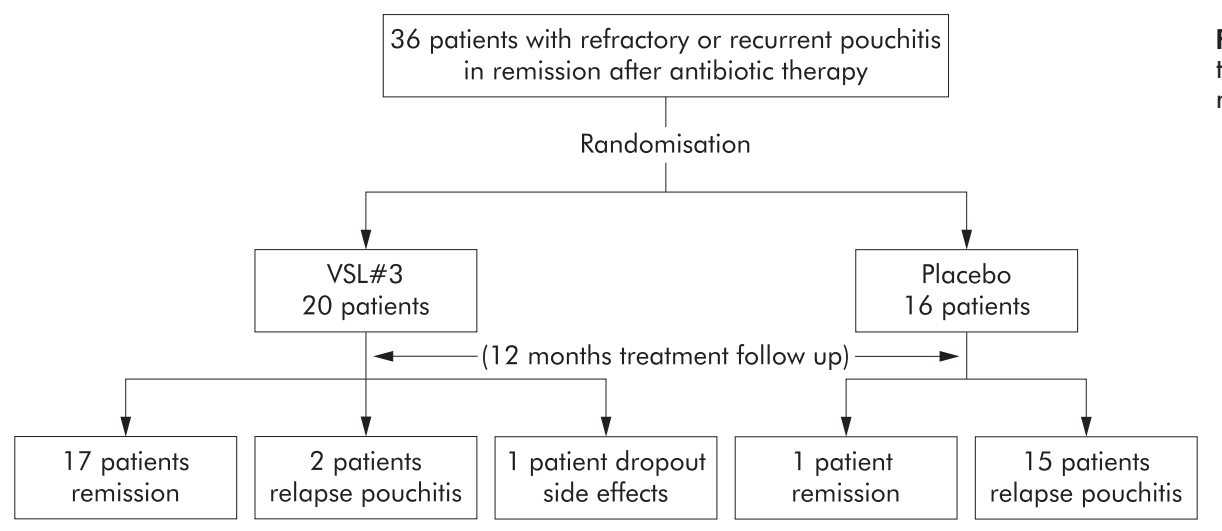

Figure 2 Study design and results of the double blind placebo controlled randomised study.

\section{Disease activity in each group}

As shown in table 3, in the 20 patients on VSL\#3, total PDAI scores were low both at entry (median 3 points) and at the time of relapse or 12 months (median 2); there was no significant difference within the group between these time points $(p=0.93)$. In the 16 patients on placebo, the total PDAI increased significantly from a median of 3 at study entry to a median of 11 at the time of relapse or 12 months $(p=0.0004)$. Similar findings were observed regarding clinical, endoscopic, and histological PDAI scores in both groups.

\section{Quality of life in each group}

As is shown in fig 4A, in the 20 patients on VSL\#3, IBDQ scores were high both at entry (median 190 points) and at the time of relapse or 12 months (median 205); this difference was not statistically significant $(p=0.3)$. In the 16 patients on placebo, IBDQ scores deteriorated significantly from a

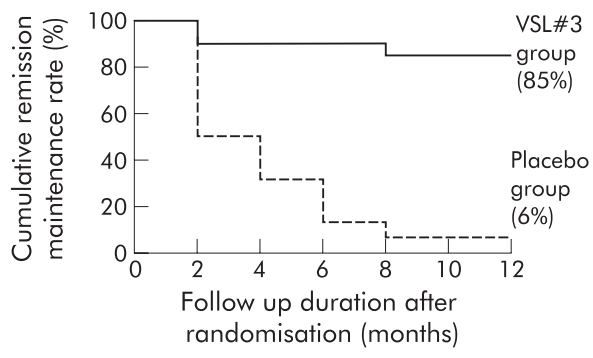

Figure 3 Cumulative remission maintenance rate in the VSL\#3 and placebo groups (Kaplan-Meier method). The cumulative remission maintenance rate after 12 months of follow up was significantly higher in the VSL\#3 group (85\%) than in the placebo group $(6 \%)(p<0.0001$, log rank test). median of 169 at study entry to a median of 105 at the time of relapse or 12 months $(p=0.0005)$ (fig $4 \mathrm{~B})$. Comparing the two groups, there was no significant difference at entry (VSL\#3 $v$ placebo: median 190 points $v 169 ; \mathrm{p}=0.42$ ) but the groups differed significantly at the time of the relapse or 12 months $(205 v 105 ; \mathrm{p}<0.0001)$.

Similar results were observed for patient satisfaction with treatment (fig 5). In the VSL\#3 group, patient satisfaction did not change significantly $(p=0.41)$ from study entry (median 4 points) to the time of relapse or 12 months (median 4 points). In the placebo group, patient satisfaction deteriorated significantly from a median of 4 at study entry to a median of 2 at the time of the relapse or 12 months $(p<0.0001)$. Comparing the two groups, there was no significant difference at entry (VSL\#3 $v$ placebo: median 4 points $v 4 ; \mathrm{p}=0.26$ ) but the groups differed significantly at the time of relapse or 12 months $(4 \vee 2 ; \mathrm{p}<0.0001)$.

\section{Stool bacterial analysis}

PCR analysis demonstrated that after administration of VSL\#3, $S$ thermophilus was detected at higher levels in all patients treated $\left(3.9 \times 10^{7} \mathrm{CFU} / \mathrm{g}\right)$ whereas only $24 \%$ and $35 \%$ of patients harboured $S$ thermophilus before probiotic treatment $\left(1.5 \times 10^{3} \mathrm{CFU} / \mathrm{g}\right)$ and after placebo treatment $\left(1.8 \times 10^{3} \mathrm{CFU} / \mathrm{g}\right)$, respectively.

VSL\#3 $B$ infantis and $B$ breve specific PCR signals were detected only after consumption of VSL\#3 whereas no amplicon was obtained amplifying colonies from the faeces of patients treated with placebo. In patients treated with the probiotic preparation, the VSL\#3 bifidobacteria, $B$ infantis and $B$ breve, were found in $53 \%$ and $67 \%$ of patients treated with VSL\#3 at a mean concentration of $2.5 \times 10^{7}$ and $4.8 \times 10^{7} \mathrm{CFU} / \mathrm{g}$ faeces, respectively. Simultaneous transient presence of both VSL\#3 $B$ infantis and B breve was observed in the faecal population of $24 \%$ of patients treated with VSL\#3. 


\begin{tabular}{|c|c|c|c|}
\hline & At study entry & $\begin{array}{l}\text { At relapse or } \\
\text { at } 12 \text { months }\end{array}$ & $\mathrm{p}$ Value \\
\hline \multicolumn{4}{|c|}{ PDAI in 20 patients on VSL\#3 } \\
\hline Clinical PDAl & $1(0-2)$ & $0(0-4)$ & 0.84 \\
\hline Endoscopic PDAI & $1(0-1)$ & $1(0-5)$ & 0.13 \\
\hline Histological PDAI & $1(0-4)$ & $1(0-3)$ & 0.25 \\
\hline Total PDAI & $3(1-7)$ & $2(0-12)$ & 0.93 \\
\hline \multicolumn{4}{|c|}{ PDAl in 16 patients on placebo } \\
\hline Clinical PDAl & $1(0-2)$ & $3(2-4)$ & 0.0003 \\
\hline Endoscopic PDAI & $1(0-1)$ & $5(3-6)$ & 0.0004 \\
\hline Histological PDAI & $1(0-2)$ & $3(1-4)$ & 0.0001 \\
\hline Total PDAI & $3(1-4)$ & $11(6-14)$ & 0.0004 \\
\hline
\end{tabular}

\section{Safety}

Of the 20 patients who received VSL\#3, one patient dropped out of the trial. This 55 year old man complained of abdominal cramps, vomiting, and diarrhoea 10 days after starting the study medication. He stopped the medication and took metronidazole $400 \mathrm{mg}$ and ciprofloxacin $500 \mathrm{mg}$ twice daily for one week, with symptom resolution. Five days later, on restarting the study drug, he developed similar symptoms, which ceased after stopping the trial medication. Two further attempts at taking the trial preparation resulted in the same symptoms. The patient had no symptoms after stopping the medication. No pathological bacteria were identified on stool culture.

No other side effects were noted in other patients. There were no significant changes in any of the laboratory values examined during and after intake of VSL\#3 or placebo.

\section{DISCUSSION}

This study has demonstrated that in patients with recurrent or refractory pouchitis who have achieved remission with
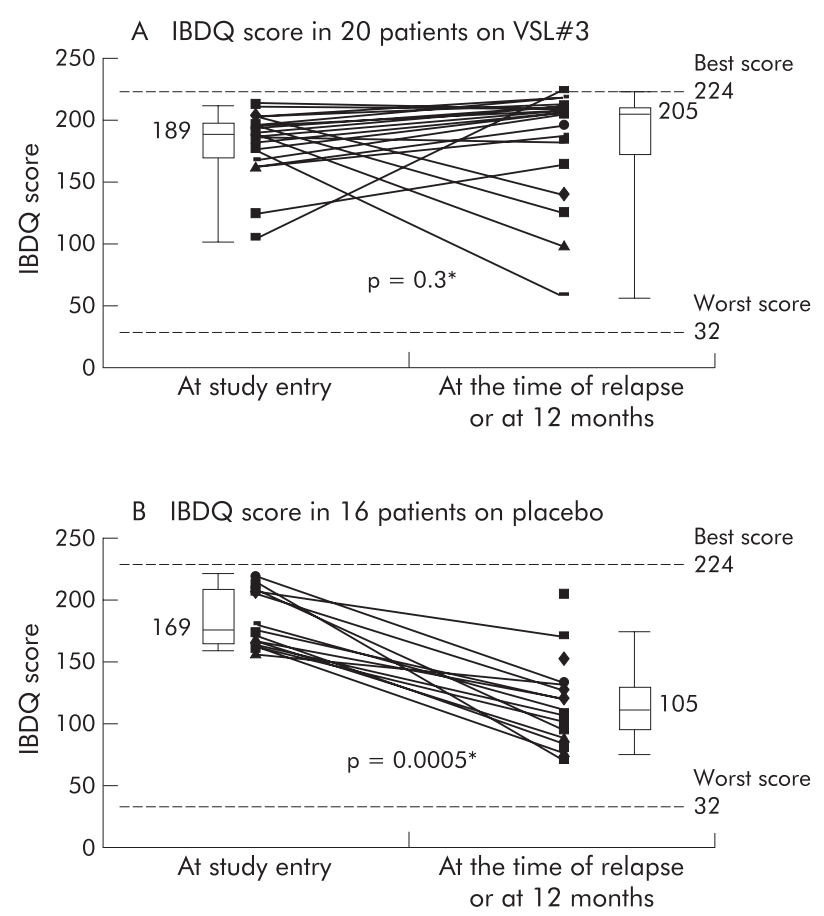

Figure 4 Inflammatory bowel disease questionnaire (IBDQ) scores at study entry and at the time of relapse or 12 months in 20 patients who received VSL\#3 (A) and in 16 patients who received placebo (B). *Wilcoxon signed rank test. intense antibiotic treatment, the probiotic therapy VSL\#3 is highly effective in maintaining remission. In parallel with clinical, endoscopic, and histological remission, a high level of QOL was maintained with this therapy.

All patients in this study had previous recurrent or refractory pouchitis and had achieved remission after a four week course of intense antibiotic therapy. We have previously reported the results of open treatment in the same cohort of patients. Antibiotic treatment would now appear to be the favoured form of treatment for active pouchitis. Alternative reported therapies include bismuth carbomer enemas, ${ }^{15}$ but in a double blind trial they were not more effective than placebo. ${ }^{16}$ Glutamine or butyrate suppositories for 21 days ${ }^{17}$ have a modest effect.

Five to $15 \%$ of patients with an ileal pouch fashioned for ulcerative colitis experience recurrent or "refractory"

\section{A Patient general satisfaction score in 20 patients on VSL\#3}

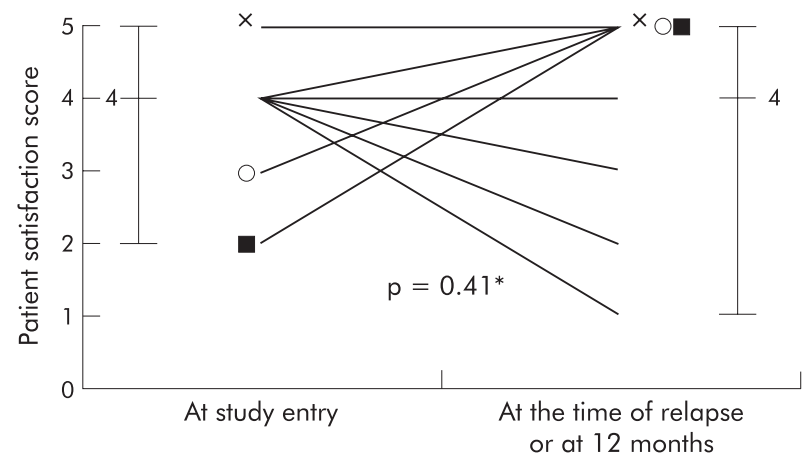

B Patient general satisfaction score in 16 patients on placebo

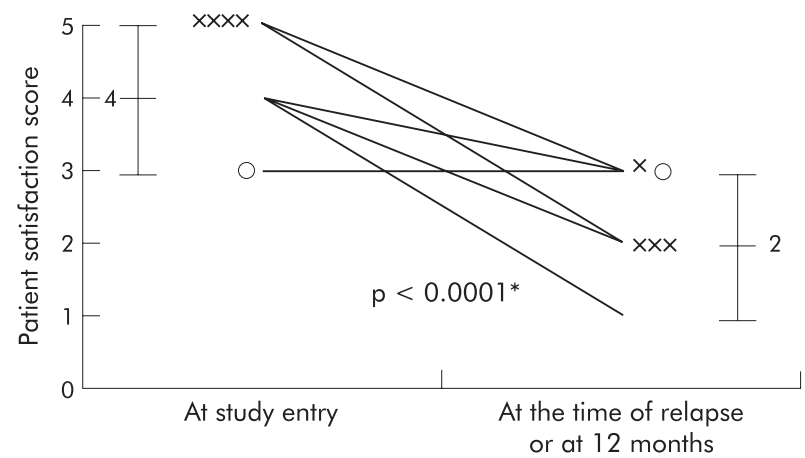

Figure 5 Patient general satisfaction scores at study entry and at the time of relapse or 12 months in 20 patients who received VSL\#3 (A) and in 16 patients who received placebo (B). *Wilcoxon signed rank test. 
pouchitis. ${ }^{3468}$ There is no uniform definition of refractory or chronic pouchitis in the literature.

Although continuous antibiotics may be able to maintain remission induced by an acute course of antibiotics, this strategy has not been formally tested. Given that an imbalance or excessive response to intraluminal bacteria seems to be involved in the pathogenesis of inflammatory bowel disease, including pouchitis, probiotic therapy to modify the bacterial flora may be an attractive option. ${ }^{18}$ Pouchitis is often regarded as a form of recurrent ulcerative colitis ${ }^{19}$ associated with an imbalance of bacterial flora within the pouch. ${ }^{20}$ Evidence for a central role of enteric bacteria in the pathogenesis is increasing, together with evidence that manipulating the flora may be an effective therapeutic strategy. For example, interleukin 10 knockout mice develop spontaneous colitis; ${ }^{21}$ under sterile conditions colitis does not develop. ${ }^{22}$ In animal models, probiotic preparations such as Lactobacillus reuteri or plantarum attenuate spontaneous colitis in interleukin 10 deficient mice ${ }^{2324}$ and chemically induced experimental colitis. ${ }^{25}{ }^{26}$ The probiotic preparation VSL\#3 ameliorates spontaneous colitis in interleukin 10 deficient mice $^{27}$ and iodoacetamide induced colitis. ${ }^{28}$

Non-pathogenic Escherichia coli have been shown to be equally effective as mesalazine in maintaining remission in ulcerative colitis. ${ }^{29}{ }^{30}$ VSL\#3 $6 \mathrm{~g} /$ day for 12 months maintained remission in 15 of 20 patients with ulcerative colitis who were allergic or intolerant to sulphasalazine or mesalamine. ${ }^{31}$

The efficacy of VSL\#3 in maintaining remission in patients with previous pouchitis has been evaluated in one previous study. Gionchetti et al ${ }^{11}$ recently demonstrated the ability of VSL\#3 to maintain remission in chronic pouchitis. Forty patients with refractory pouchitis were initially treated with a combination of ciprofloxacin and rifaximin for one month. After induction of remission, 20 patients were randomised to receive VSL\#3 $3 \mathrm{~g}$ twice per day, and 20 patients received placebo, for nine months or until relapse. Seventeen patients on VSL\#3 remained in remission while all on placebo relapsed.

The current study has confirmed the effectiveness of VSL\#3 as a maintenance treatment in patients with recurrent or refractory pouchitis. Four aspects of the current study extend the observations previously made by Gionchetti and colleagues. ${ }^{11}$ Firstly, metronidazole was used instead of rifaximin because metronidazole has been demonstrated in double blind studies to be an effective treatment for active pouchitis. ${ }^{9}$ Metronidazole is also more widely available than rifaximin. Secondly, in this study, VSL\#3 was taken as a single $6 \mathrm{~g}$ daily dose, instead of $3 \mathrm{~g}$ twice daily. It was our intention to simplify treatment as much as possible. We also hypothesised that this dosing regimen may allow greater residence time and higher bacterial concentration in the gut. Patients usually empty their pouch repeatedly during the day. Thirdly, we extended the study period from nine to 12 months. Lastly, we have demonstrated that QOL and patient satisfaction is enhanced by probiotic therapy, in parallel with its clinical efficacy.

Despite the change in initial antibiotic and probiotic regimens, and longer observation, the current study has produced similar results. Maintenance remission rates were $85 \%$ and $85 \%$ in the VSL\#3 group and $6 \%$ and $0 \%$ in the placebo group in the current study and in the study of Gionchetti and colleagues, ${ }^{11}$ respectively.

In addition to maintaining remission, this probiotic mixture has been shown to prevent the development of pouchitis after pouch formation. ${ }^{32}$

Some potential mechanisms for the beneficial effect of VSL\#3 in inflammatory bowel disease and pouchitis have been elucidated. Ulisse and colleagues ${ }^{33}$ have reported increased tissue levels of tumour necrosis factor $\alpha$, interferon $\gamma$, inducible nitric oxide synthase, and matrix metalloproteinases 2 and 9 in pouchitis, all of which were reduced after treatment by antibiotics followed by VSL\#3. In that study, and previously, ${ }^{34}$ increased tissue interleukin 10 was demonstrated following probiotic therapy.

Madsen and colleagues ${ }^{27}$ demonstrated that treatment of interleukin 10 gene deficient mice with VSL\#3 results in normalisation of colonic physiological function and barrier integrity in conjunction with a reduction in mucosal secretion of tumour necrosis factor $\alpha$ and interferon $\gamma$. They have also shown in an in vitro study that epithelial barrier function is enhanced by exposure to a proteinaceous soluble factor secreted by the bacteria in VSL\#3.

In conclusion, the probiotic mixture VSL\#3 is effective as a maintenance treatment in patients with recurrent or refractory pouchitis. This is achieved with minimal side effects.

\section{ACKNOWLEDGEMENT}

This study was partially supported by VSL Pharmaceuticals Inc., Fort Lauderdale, USA.

\section{Authors' affiliations}

T Mimura, I C Talbot, R J Nicholls, M A Kamm, Departments of Medicine, Surgery, and Pathology, St Mark's Hospital, London, UK F Rizzello, U Helwig, G Poggioli, P Gionchetti, M Campieri, Departments of Internal Medicine, Gastroenterology, and Surgery, University of Bologna, Bologna, Italy

S Schreiber, First Department of Medicine, Christian-AlbrechtsUniversity, Kiel, Germany

\section{REFERENCES}

1 Melville DM, Ritchie JK, Nicholls RJ, et al. Surgery for ulcerative colitis in the era of the pouch: the St Mark's Hospital experience. Gut 1994;35:1076-180.

2 Jimmo B, Hyman NH. Is ileal pouch-anal anastomosis really the procedure of choice for patients with ulcerative colitis? Dis Colon Rectum 1998;41:41-5.

3 Setti-Carraro P, Ritchie JK, Wilkinson KH, et al. The first 10 years' experience of restorative proctocolectomy for ulcerative colitis. Gut 1994;35:1070-5.

4 Stahlberg D, Gullberg K, Liljeqvist L, et al. Pouchitis following pelvic pouch operation for ulcerative colitis. Incidence, cumulative risk, and risk factors. Dis Colon Rectum 1996;39:1012-18.

5 Romanos J, Samarasekera DN, Stebbing JF, et al. Outcome of 200 restorative proctocolectomy operations: the John Radcliffe Hospital experience. $\mathrm{Br} J$ Surg 1997;84:814-18.

6 Keranen $U$, Luukkonen $P$, Jarvinen $H$. Functional results after restorative proctocolectomy complicated by pouchitis. Dis Colon Rectum 1997:40:764-9.

7 Meagher AP, Farouk R, Dozois RR, et al. J ileal pouch-anal anastomosis for chronic ulcerative colitis: complications and long-term outcome in 1310 patients. Br J Surg 1998;85:800-3.

8 Hurst RD, Chung TP, Rubin M, et al. The implications of acute pouchitis on the long-term functional results after restorative proctocolectomy. Inflamm Bowel Dis 1998;4:280-4.

9 Madden MV, Mclntyre AS, Nicholls RJ. Double-blind crossover trial of metronidazole versus placebo in chronic unremitting pouchitis. Dig Dis Sci 1994:39:1193-6.

10 Mimura T, Rizzello F, Helwig U, et al. Four week open label trial of metronidazole and ciprofloxacin for the treatment of recurrent or refractory pouchitis. Aliment Pharmacol Ther 2002;16:909-17.

11 Gionchetti P, Rizzello F, Venturi A, et al. Oral bacteriotherapy as maintenance treatment in patients with chronic pouchitis: a double-blind, placebocontrolled trial. Gastroenterology 2000;1 19:305-9.

12 Guyatt G, Mitchell A, Irvine EJ, et al. A new measure of health status for clinical trials in inflammatory bowel disease. Gastroenterology 1989:96:804-10.

13 de Boer AG, Wijker W, Bartelsman JF, et al. Inflammatory bowel disease questionnaire: cross-cultural adaptation and further validation. Eur J Gastroenterol Hepatol 1995;7:1043-50.

14 Sandborn WJ, Tremaine WJ, Batts KP, et al. Pouchitis after ileal pouch-anal anastomosis: a pouchitis disease activity index. Mayo Clin Proc 1994;69:409-15

15 Gionchetti P, Rizzello F, Venturi A, et al. Long-term efficacy of bismuth carbomer enemas in patients with treatment-resistant chronic pouchitis. Aliment Pharmacol Ther 1997;11:673-8.

16 Tremaine WJ, Sandborn WJ, Wolff BG, et al. Bismuth carbomer foam enemas for active chronic pouchitis: a randomized, double-blind, placebo-controlled trial. Aliment Pharmacol Ther 1997;1 1:1041-6.

17 Wischmeyer P, Pemberton JH, Phillips SF. Chronic pouchitis after ileal pouchanal anastomosis: responses to butyrate and glutamine suppositories in a pilot study. Mayo Clin Proc 1993;68:978-81. 
18 Mimura T, Kaminishi M, Kamm MA. Probiotics for pouchitis and its endoscopic findings. Dig Endosc 2002;14:47-52.

19 Luukkonen P, Jarvinen $\mathrm{H}$, Tanskanen $M$, et al. Pouchitis-recurrence of the inflammatory bowel disease? Gut 1994:35:243-6.

20 Ruseler-Van Embden JG, Schouten WR, van Lieshout LM. Pouchitis: result of microbial imbalance? Gut 1994;35:658-64.

21 Kuhn R, Lohler J, Rennick D, et al. Interleukin-10-deficient mice develop chronic enterocolitis. Cell 1993;75:263-74.

22 Sellon RK, Tonkonogy S, Schultz M, et al. Resident enteric bacteria are necessary for development of spontaneous colitis and immune system activation in interleukin-10-deficient mice. Infect Immun system activation in

23 Schultz M, Veltkamp C, Dieleman LA, et al. Lactobacillus plantarum 299Vs in the treatment and prevention of spontaneous colitis in interleukin-10- deficient mice. Inflamm Bowel Dis 2002:8:71-80.

24 Madsen KL, Doyle JS, Jewell LD, et al. Lactobacillus species prevents colitis in interleukin 10 gene-deficient mice. Gastroenterology 1999; 116:1107-14.

25 Fabia R, Ar'Rajab A, Johansson ML, et al. The effect of exogenous administration of Lactobacillus reuteri R2LC and oat fiber on acetic acidinduced colitis in the rat. Scand J Gastroenterol 1993;28:155-62.

26 Mao Y, Nobaek S, Kasravi B, et al. The effects of Lactobacillus strains and oat fiber on methotrexate-induced enterocolitis in rats. Gastroenterology $1996 ; 111: 334-44$.
27 Madsen K, Cornish A, Soper $\mathrm{P}$, et al. Probiotic bacteria enhance murine and human intestinal epithelial barrier function. Gastroenterology $2001 ; 121: 580-91$

28 Shibolet O, Karmeli F, Eliakim R, et al. Probiotics ameliorates iodoacetamideinduced colitis. Gastroenterology 1999;116:A818.

29 Kruis W, Schutz E, Fric P, et al. Double-blind comparison of an oral Escherichia coli preparation and mesalazine in maintaining remission of ulcerative colitis. Aliment Pharmacol Ther 1997;11:853-8.

30 Rembacken BJ, Snelling AM, Hawkey PM, et al. Non-pathogenic Escherichia coli versus mesalazine for the treatment of ulcerative colitis: a randomised trial. Lancet 1999;354:635-9.

31 Venturi A, Gionchetti P, Rizzello F, et al. Impact on the composition of the faecal flora by a new probiotic preparation: preliminary data on maintenance treatment of patients with ulcerative colitis. Aliment Pharmacol Ther 1999;13:1103-8.

32 Gionchetti P, Rizzello F, Venturi A, et al. Prophylaxis of pouchitis onset with probiotic therapy: A double-blind, placebo controlled trial. Gastroenterology 2000;118:A190.

33 Ulisse S, Gionchetti P, D'Alo S, et al. Expression of cytokines, inducible nitric oxide synthase, and matrix metalloproteinases in pouchitis: effects of probiotic treatment. Am J Gastroenterol $2001 ; 96: 2691-9$.

34 Gionchetti P, Rizzello F, Cifone G, et al. In vivo effect of a highly concentrated probiotics on IL-10 pelvic-pouch tissue levels. Gastroenterology 1999;116:A723. 